\title{
EL PATRIMONIO CULTURAL DESDE LA PERSPECTIVA DE LA ORGANIZACIÓN MUNDIAL DEL TURISMO
}

\author{
CULTURAL HERITAGE FROM THE PERSPECTIVE OF THE WORLD \\ TOURISM ORGANIZATION
}

\author{
Juan Carlos Paredes Izquierdo \\ Universidad de San Martín de Porres, Perú
}

Aceptado: 7 de setiembre de 2018

\section{RESUMEN}

Se plantea como objetivo contribuir a tender puentes de diálogo entre el sector cultural y el sector turístico, específicamente, entre los profesionales e instituciones encargados de la conservación y gestión del patrimonio cultural, y los profesionales e instituciones encargados de la gestión turística. Para este fin, se busca demostrar la importancia que la Organización Mundial del Turismo otorga al patrimonio cultural, no solo como recurso turístico, sino como elemento fundamental para la identidad de las comunidades y para el logro de un desarrollo sostenible, en concordancia con los Objetivos de Desarrollo Sostenible de las Naciones Unidas. Para lograrlo, se recurre a diversas fuentes, documentos e informes que permiten mostrar esta óptica particular desde el campo del turismo.

Palabras clave: patrimonio cultural, patrimonio inmaterial, turismo cultural, Organización Mundial del Turismo, Código Ético Mundial para el Turismo.

\begin{abstract}
The purpose of this paper is to help build bridges of dialogue between the cultural sector and the tourism sector, or more specifically, between professionals and institutions responsible for the conservation and management of cultural heritage, and professionals and institutions responsible for tourism management. To this end, it seeks to demonstrate the importance that the World Tourism Organization grants to cultural heritage, not only as a tourist resource, but also as a fundamental element for the identity of communities and for the achievement of a sustainable development, in accordance with the United Nations Sustainable Development Goals. To attain this, we use various sources, documents and reports that show this particular perspective from the field of tourism.
\end{abstract}

Keywords: cultural heritage, intangible heritage, cultural tourism, World Tourism Organization, Global Code of Ethics for Tourism.

Este es un artículo Open Access bajo la licencia Creative Commons AtribuciónNoComercial-Compartirlgual 4.0

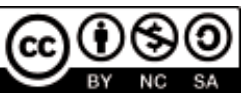




\section{Introducción}

Como sucede con todos los objetos de análisis, el patrimonio cultural puede mirarse desde diversas ópticas y perspectivas, ya sea desde la de los investigadores, la de los gestores o la de la comunidad; como también desde la del arqueólogo, del economista o del experto en turismo, solo por mencionar algunos ejemplos. Dependiendo de la posición del observador, del 'cristal' con el que se observa o del 'para qué' de la observación, a veces estas ópticas y perspectivas encuentran puntos en común y se alinean con facilidad. Otras veces, en cambio, parecieran estar contrapuestas y en permanente confrontación.

Un campo en el que suele apreciarse esto último es el del uso turístico del patrimonio cultural. En este campo, es común el enfrentamiento, por un lado, de los profesionales del estudio y conservación del patrimonio (arqueólogos, antropólogos, historiadores, arquitectos, entre otros), y por el otro, el de los profesionales del turismo. Este enfrentamiento se lleva muchas veces al sector público, en el que los ministerios de cultura o las entidades que ejercen dicha función, se enfrentan a los ministerios de turismo o las entidades que ejerce tal función. Si bien esto no sucede en todos los casos, es evidente que se hace necesario establecer los espacios para un diálogo abierto, transparente y respetuoso entre ambos frentes, en el que se favorezcan los enfoques multi y transdisciplinarios, con el fin de construir propuestas y soluciones de tipo win-win o ganar-ganar, es decir en las que todas las partes involucradas se vean beneficiadas.

Para lograr este fin, es necesario que las partes involucradas, se acerquen mutuamente unas a otras, con la intención de conocer y comprender sus fines, sus intereses, sus objetivos y el marco conceptual desde el que diseñan sus planteamientos y operan. Una profesión que existe desde hace mucho pero que recientemente se ha formalizado y que parte desde este enfoque, es precisamente la Gestión Cultural.

El presente estudio se aproxima a este problema desde el análisis del enfoque que plantea la Organización Mundial del Turismo - OMT (UNWTO en inglés), en torno al patrimonio cultural, a su significado y valoración, a su conservación y salvaguarda, encuadradas en las posibilidades y retos de su uso turístico, con el objetivo de mostrar las ventajas de entender al turismo y a la conservación del patrimonio no como dos actividad opuestas y en conflicto, sino como dos actividades complementarias que tienen fines compartidos.

\section{La cultura, el patrimonio cultural, el turismo y la OMT}

La Organización Mundial del Turismo (OMT) es la agencia de las Naciones Unidas encargada de la promoción de un turismo responsable, sostenible y universalmente accesible. Sus 159 países miembros y más de 500 miembros afiliados, la convierten en la principal organización internacional en el ámbito turístico.

El patrimonio cultural es un tema de permanente interés para la OMT. La relación entre ambos resulta obvia si se tiene en cuenta que en la actualidad, la cultura y sus diversas expresiones, constituyen uno de los principales atractivos con los que cuentan los destinos turísticos. Al turismo orientado hacia estas expresiones se le denomina 'turismo cultural'. 
Una definición oficial y de consenso sobre el turismo cultural, es la que se consigna en las actas de la $22^{\circ}$ Sesión de la Asamblea General de la OMT:

Cultural tourism is a type of tourism activity in which the visitor's essential motivation is to learn, discover, experience and consume the tangible and intangible cultural attractions/products in a tourism destination. These attractions/products relate to a set of distinctive material, intellectual, spiritual and emotional features of a society that encompasses arts and architecture, historical and cultural heritage, culinary heritage, literature, music, creative industries and the living cultures with their lifestyles, value systems, beliefs and traditions. (World Tourism Organization, 2017)

Esta definición recoge las diversas formas a través de las cuales se expresa la cultura en sus manifestaciones tangibles e intangibles, tal como pueden ser apreciadas, disfrutadas o ‘consumidas’ por los turistas.

Si bien el fenómeno del turismo cultural ya se había identificado desde algunas décadas atrás, tomó algún tiempo identificar sus causas, efectos e implicancias. Por ejemplo en 1999, una conocida presentación titulada The power of cultural tourism (Lord, 1999) ya mencionaba entre las causas del desarrollo del turismo cultural en los Estados Unidos, los cada vez más altos niveles educativos de la población, el incremento en la edad de las poblaciones, el cada vez más importante rol económico de la mujer, el incremento de los viajes de corta duración, y el interés creciente en la conservación y la sostenibilidad, entre otros. Un elemento fundamental en este proceso, que aún no se expresaba con todo su poder en 1999, ha sido la explosión de la información escrita y audiovisual, producida, distribuida y consumida a través de los medios digitales y mediante los dispositivos portátiles.

Estudios posteriores como The impact of culture on tourism, confirmaron estas tendencias y añadieron otras, tales como el interés creciente en la cultura como fuente de identidad y diferenciación para enfrentar la globalización, los estilos de consumo postmodernos orientados menos a lo material y más al desarrollo personal, o el deseo de ‘vivir experiencias’ (Organisation for Economic Cooperation and Development, 2009).

En cuanto a las cifras del turismo cultural, el informe Tourism and culture synergies, señala un método amplio y otro restringido de entender y medir el turismo cultural a nivel global. Según el método restringido, son turistas culturales aquellos que viajan motivados por la cultura del lugar que van a visitar; mientras que según el método amplio, lo son aquellos que sin haber viajado como consecuencia de una motivación cultural, una vez que llegan a su destino, realizan actividades culturales, como por ejemplo, visitar un museo o un sitio arqueológico. De la cifra estimada de arribos internacionales de turistas en el año 2015 estimada por la OMT en 1184 millones, el porcentaje de turistas culturales asciende al $16 \%$ si se sigue el método restringido, y a $47 \%$ si se sigue el método amplio (World Tourism Organization, 2018a).

En el Perú, la información sobre este dato proviene del Perfil del Turista Extranjero (Comisión de Promoción del Perú para la Exportación y el Turismo, 2017). En este caso y aplicando el método amplio, del total de 4 millones de turistas, el 91\% fueron turistas culturales, lo que convierte al país en un destino netamente cultural. 
No obstante esta realidad, el interés de la OMT en el patrimonio cultural no se agota, ni mucho menos, en este fin utilitario. Para comprender esto, es necesario remitirnos en primer lugar a los fines, objetivos y prioridades de la OMT. En este sentido, debe recordarse que la OMT forma parte del sistema de las Naciones Unidas, y que por lo tanto comparte sus objetivos, entre ellos los Objetivos de Desarrollo Sostenible (ODS), pero orienta su acción desde y hacia el campo específico del turismo. Los ODS forman parte de la Agenda 2030, constituyéndose en 17 objetivos que orientan los esfuerzos de los países para lograr un mundo sostenible en el año 2030. Es ese contexto que deben entenderse los fines, objetivos principales y prioridades de la OMT, los cuales están estrechamente relacionados con el tema del presente estudio, pues hacen referencia a valores y principios que están en el corazón de la naturaleza y los objetivos de la conservación y la salvaguarda del patrimonio cultural, tales como el respeto a la diversidad, los derechos humanos, la ética y el desarrollo sostenible, entre otros.

Empezando con sus fines, la OMT señala como su fin principal:

La promoción y desarrollo del turismo con vistas a contribuir al desarrollo económico, la comprensión internacional, la paz, la prosperidad y el respeto universal, y la observancia de los derechos humanos y las libertades fundamentales para todos, sin distinción de raza, sexo, lengua o religión. (World Tourism Organization, 2015a, p. 15)

En cuanto a sus objetivos principales, resaltan los de contribuir a que la actividad se convierta en instrumento de crecimiento económico, desarrollo inclusivo y sostenibilidad ambiental, a través de la aplicación del Código Ético Mundial para el Turismo; y su compromiso con el logro de los Objetivos de Desarrollo Sostenible (ODS) de la Agenda 2030, orientados a reducir la pobreza y a fomentar el desarrollo sostenible en todo el mundo (World Tourism Organization, 2016). Precisamente, el objetivo 11.4 de los ODS señala la necesidad de "redoblar los esfuerzos para proteger y salvaguardar el patrimonio cultural y natural del mundo” (Naciones Unidas, s.f.).

De igual manera, con relación a las prioridades de la OMT, destacan nuevamente la promoción del desarrollo sostenible del turismo y el impulso a la contribución del turismo a la reducción de la pobreza y al desarrollo. (Organización Mundial del Turismo, 2016). Finalmente, y recurriendo a un documento más reciente, la Declaración de Chengdu sobre el turismo y los Objetivos de Desarrollo Sostenible, señala la importancia del turismo como un instrumento que permite contribuir a la protección del patrimonio cultural (Organización Mundial del Turismo, 2017).

\section{El Código Ético Mundial para el Turismo}

Uno de los instrumentos rectores más importantes de la OMT es el Código Ético Mundial para el Turismo. Se trata de un conjunto de principios "dirigido a gobiernos, empresas turísticas, comunidades y turistas por igual”, con el objetivo de "ayudar a maximizar los beneficios del sector, minimizando a la vez sus posibles consecuencias negativas para el medio ambiente, el patrimonio cultural y las sociedades de alrededor del mundo”. (Organización Mundial del Turismo, s.f.). El Código fue aprobado por la Asamblea General de la OMT en 1999 y fue reconocido dos años más tarde por las Naciones Unidas. Si bien no es un instrumento jurídicamente 
vinculante, sí contempla un mecanismo de aplicación voluntaria. El documento consta de diez artículos, los cuales constituyen principios rectores de la actividad turística y sus implicancias en los ámbitos económico, social, cultural y ambiental.

La cultura, la diversidad cultural, el patrimonio cultural y la importancia y necesidad de su conservación, son temas presentes a lo largo de diferentes artículos del texto, lo que constituye una muestra de la importancia que la OMT les otorga. Sin embargo, son dos artículos en los que el tema se aborda con precisión. Se trata de los artículos 1 (incisos 4 y 5) y 4 (incisos 1, 2, 3, 4) que reproducimos a continuación.

Art. 1 Contribución del turismo al entendimiento y al respeto mutuos entre hombres y sociedades.

4. Los atentados, agresiones, secuestros o amenazas dirigidos contra turistas o trabajadores del sector turístico, así como la destrucción intencionada de instalaciones turísticas o de elementos del patrimonio cultural o natural, de conformidad con la legislación nacional respectiva deben condenarse y reprimirse con severidad.

5. En sus desplazamientos, los turistas y visitantes evitarán todo acto criminal o considerado delictivo por las leyes del país que visiten, y cualquier comportamiento que pueda resultar chocante o hiriente para la población local, o dañar el entorno del lugar. Se abstendrán de cualquier tipo de tráfico de drogas, armas, antigüedades, especies protegidas, y productos y sustancias peligrosas o prohibidas por las reglamentaciones nacionales. (Organización Mundial del Turismo, 2001, p. 4)

Desde su primer artículo, el Código resalta una de las principales funciones que se le asigna al turismo, como actividad que puede contribuir al entendimiento mutuo entre hombres y sociedades, lo que inmediatamente nos refiere al respeto por la cultura local de los destinos y consecuentemente por su patrimonio cultural. El artículo es más específico en sus incisos cuando señala la necesidad de reprimir la destrucción del patrimonio cultural (inc. 4), y el tráfico de antigüedades (inc. 5). En ambos casos referidos principalmente al patrimonio tangible o material, se señala la necesidad de que se aplique la legislación nacional.

Siguiendo con el análisis de este instrumento, el Código incorpora otro artículo, esta vez dedicado exclusivamente al tema, en el que aborda no solo los riesgos a los que podría exponerse el patrimonio material, sino también el intangible o inmaterial (que es el término empleado por la UNESCO en la Convención para la Salvaguardia del Patrimonio Cultural Inmaterial del año 2003).

Art. 4 El turismo, factor de aprovechamiento y enriquecimiento del patrimonio cultural de la humanidad

1. Los recursos turísticos pertenecen al patrimonio común de la humanidad. Las comunidades en cuyo territorio se encuentran tienen con respecto a ellos derechos y obligaciones particulares.

2. Las políticas y actividades turísticas se llevarán a cabo con respeto al patrimonio artístico, arqueológico y cultural, que deben proteger y transmitir a las generaciones futuras. Se concederá particular atención a la protección y a la rehabilitación de los monumentos, santuarios y museos, así como de los lugares de interés histórico o arqueológico, que deben estar ampliamente abiertos 
a la frecuentación turística. Se fomentará el acceso del público a los bienes y monumentos culturales de propiedad privada con todo respeto a los derechos de sus propietarios, así como a los edificios religiosos sin perjuicio de las necesidades del culto.

3. Los recursos procedentes de la frecuentación de los sitios y monumentos de interés cultural habrían de asignarse preferentemente, al menos en parte, al mantenimiento, a la protección, a la mejora y al enriquecimiento de ese patrimonio.

4. La actividad turística se organizará de modo que permita la supervivencia y el florecimiento de la producción cultural y artesanal tradicional, así como del folklore, y que no conduzca a su estandarización y empobrecimiento. (Organización Mundial del Turismo, 2001, p. 5)

Aunque es clara la intención de la OMT en cuanto a resaltar la importancia del patrimonio cultural y su conservación, llama la atención las palabras elegidas para la redacción de texto inicial de este artículo, especialmente la expresión “enriquecimiento del patrimonio cultural de la humanidad”. El inciso 3 repite la expresión y le agrega la palabra "mejora”, pero lo hace dentro de un contexto que de alguna manera permite inferir que está referido a su conservación y puesta en valor. De igual manera, cuando en el texto inicial se refiere exclusivamente al patrimonio cultural de la humanidad, podría inferirse que se está haciendo referencia a aquellas expresiones incluidas en la Lista del Patrimonio Mundial de la UNESCO, pero cuando en el inciso 1 señala: "Los recursos turísticos pertenecen al patrimonio común de la humanidad”, se entiende que no se refiere exclusivamente a esta lista, sino que resalta de manera extensa el valor que tiene el patrimonio cultural para todos los seres humanos.

Dos ideas particularmente importantes mencionadas en ese artículo, son las referidas a la necesidad de que los ingresos generados por la actividad turística como consecuencia de las visitas de los turistas, sean destinados preferentemente a la conservación y puesta en valor del bien patrimonial; y a la necesidad de evitar que el turismo conduzca a la estandarización y empobrecimiento del patrimonio inmaterial en los destinos. Estos temas son especialmente importantes pues en la práctica es común ver en diversos países y también en el Perú, que muchos bienes patrimoniales se encuentran desatendidos o que no se invierte lo suficiente en su conservación, pese a que son generadores de ingresos importantes generados por las visitas de los turistas. De igual manera, es lamentable comprobar que en otros casos en los que la actividad turística se lleva a cabo de manera espontánea y sin ningún control, la presencia de los turistas causa un impacto en las costumbres, modos de vida y otras expresiones culturales locales, en parte por la presencia misma de otras culturas, pero principalmente por el afán de las comunidades de adecuar sus expresiones culturales a las necesidades y requerimientos de los turistas, con el objetivo de incrementar sus ingresos en el corto plazo. Esta práctica no solo tiene un impacto negativo en la cultura local, sino que, al perder la autenticidad que constituye su principal atractivo, produce a largo plazo un efecto económico contrario al deseado. 


\section{El patrimonio cultural en otros documentos de la OMT}

La importancia y el valor que la OMT le asigna al patrimonio cultural como elemento fundamental de la identidad de las comunidades, pero también como objeto de un potencial uso turístico, y por lo tanto generador de oportunidades para el desarrollo socioeconómico de los destinos, se ven reflejados también en otros documentos e informes que abordan estas cuestiones desde perspectivas específicas. Tal es el caso del Communicating Heritage. A Handbook for the Tourism Sector, publicación en la cual la OMT plantea un escenario en el que la noción de patrimonio se encuentra en evolución permanente y en el que es necesario resaltar el potencial de unos turistas bien informados y sensibles, que contribuyan a impulsar los esfuerzos de conservación y la toma de conciencia del público (World Tourism Organization, 2011).

Otro ejemplo es el del Global Report on Cultural Routes and Itineraries, informe sobre las rutas culturales, en el que se hace hincapié en que un tema fundamental en el proceso de 'conversión' de un bien patrimonial en un producto turístico, no se haga en un vacío, sino como parte de un conjunto de actividades de gestión (conservación, generación de conocimientos, puesta en valor y socialización, entre otros) (World Tourism Organization, 2015b). Siguiendo esa línea, en Tourism and culture synergies, documento al que ya se ha hecho referencia con anterioridad, la OMT se explaya en relación a todos los vínculos e interacciones que surgen a partir del uso turístico de las expresiones culturales y del patrimonio, y que han sido identificados mediante la aplicación de una encuesta realizada en 69 de los 156 Estados miembros de la organización (World Tourism Organization, 2018a). Es de especial interés, en este estudio, el análisis que se hace sobre la conceptualización misma del turismo cultural, en un contexto en el que esta modalidad se encuentra en expansión y amenaza con saturar ciertos destinos y poner en riesgo la existencia misma de ciertos monumentos. El tema de la saturación turística que hoy en día se asocia al término ‘turismofobia', ya había sido analizado anteriormente en Gestión de la saturación turística en sitios de interés natural y cultural. Guía práctica (Organización Mundial del Turismo, 2004).

Como puede apreciarse, el valor especial de muchos de los documentos de la OMT que han sido revisados para el presente estudio, radica en que permiten difundir entre los profesionales y gestores turísticos, diversos conceptos de importancia fundamental relacionados con el patrimonio cultural y su conservación, relacionándolos con casos concretos en distintos continentes. Un documento de muy especial interés, que es ejemplo de esto, es el Turismo y patrimonio cultural inmaterial. En este texto, la OMT aborda el concepto de patrimonio cultural inmaterial y propone una guía para los funcionarios y gestores del sector turístico para el desarrollo de sus actividades, considerando la salvaguarda de este patrimonio (World Tourism Organization, 2013a).

También viene al caso mencionar el informe Tourism at World Heritage Sites. Challenges and opportunities, fruto del Seminario Internacional en Turismo llevado a cabo en Izmir, Turquía, en el año 2013, y en el que se resalta la importancia crucial de una comunicación efectiva de los mensajes, significados y valores subyacentes en las expresiones culturales constituidas en bienes patrimoniales orientada a sensibilizar a los turistas y a la población, con el fin de contribuir a su conservación (World Tourism Organization, 2013b).

En cuanto a las referencias más recientes que demuestran la importancia que la OMT le otorga al patrimonio cultural, debe mencionarse Tourism and Development, que tanto en su marco teórico del primer tomo, como 
en el estudio de casos del segundo, incluyen al patrimonio cultural como uno de los pilares del desarrollo, junto con los valores culturales y la diversidad, y admiten el riesgo potencial que puede representar el turismo cuando, siguiendo lo expresado en un informe de la UNESCO, se señala que:

In the process of standardizing, modifying and commodifying cultural assets for use in cultural tourism there is a serious risk of loss of authenticity. The problem is that too often the 'packaging and presentation' of heritage is carried out by the tourism industry for the benefit of its members and not by those responsible for the safeguarding of cultural heritage. As a result, both the physical fabric of a heritage property and its intangible aspects are trivialized and compromised. (World Tourism Organization, 2018b, p. 83)

La referencia a esta cita de la UNESCO y el reconocimiento de la responsabilidad que le corresponde asumir a los gestores turísticos, es a nuestro criterio, un ejemplo a seguir si se pretende crear lazos constructivos y sostenibles entre el sector turístico y el de la cultura y el patrimonio.

Finalmente y para el caso del Perú, se cuenta con un documento específico elaborado por la OMT. Se trata del informe Alianza entre turismo y cultura en el Perú - Modelos de colaboración entre turismo, cultura y comunidad, que presenta la riqueza patrimonial material e inmaterial del país, incluyendo con detalle los sitios incluidos en la Lista del Patrimonio Mundial de la UNESCO, así como las manifestaciones de su patrimonio inmaterial, expresadas en fiestas, cocinas y rutas culturales, antes de plantear las posibilidades de su uso y aprovechamiento turístico (Organización Mundial del Turismo, 2016).

\section{Conclusiones}

La conservación eficaz del patrimonio cultural pasa necesariamente por la coordinación de los esfuerzos de todos los agentes y sectores involucrados en su gestión y aprovechamiento, concretamente a aquellos vinculados con su salvaguarda y a aquellos vinculados al turismo. Esta coordinación de esfuerzos solo puede llevarse a cabo partiendo de un proceso de diálogo en el que las partes involucradas expresen sus intereses y necesidades, pero a la vez reconozcan sus responsabilidades y conflictos. Una guía fundamental y de gran utilidad para este proceso, está constituida por los Objetivos de Desarrollo Sostenible de las Naciones Unidas. Del análisis efectuado en el presente estudio, se concluye que la Organización Mundial del Turismo viene desarrollando, desde hace varios años, un esfuerzo sostenido por contribuir desde su sector a la conservación del patrimonio cultural de las localidades. La evidencia de esto puede encontrarse en los diversos documentos estudiados en los que no solo desarrolla su visión y marco conceptual sobre el patrimonio, sino también la gran cantidad de casos en los que esto se ha hecho tangible. Este es sin duda un esfuerzo que debe ser imitado por los gestores turísticos a escala nacional y local, y también por extensión, por los gestores del patrimonio desde sus propios marcos teóricos, normativos y de acción. 


\section{Referencias}

Comisión de Promoción del Perú para la Exportación y el Turismo. (2017). Perfil del Turista Extranjero 2017. PromPerú. Recuperado de https:/www.promperu.gob.pe/TurismoIN/sitio/VisorDocumentos?titulo=Perfil\%20del\%20Turista\%20Extranjero\%202017\&url= / Uploads/perfiles_extranjeros/40/PTE2017.pdf\&nombObjeto=PerfTuristaExt\&back=/TurismoIN/sitio/PerfTuristaExt\&issuuid=

Lord, G. (1999). The power of cultural tourism. Lord Cultural Resources Planning and Management. Recuperado de https://www.lord.ca/ Media/Artcl_PowerCulturalTourism-GL.pdf

Naciones Unidas (s.f.). Objetivos de Desarrollo Sostenible. Recuperado de https://www.un.org/sustainabledevelopment/es/

Organisation for Economic Cooperation and Development. (2009). The impact of culture on tourism, OECD. Recuperado de https:// read.oecd-ilibrary.org/industry-and-services/the-impact-of-culture-on-tourism_9789264040731-en\#page3

Organización Mundial del Turismo. (2001). Código Ético Mundial para el Turismo. OMT. Recuperado de http://cf.cdn.unwto.org/sites/ all/files/docpdf/gcetbrochureglobalcodees.pdf

Organización Mundial del Turismo. (2004). Gestión de la saturación turística en sitios de interés natural y cultural. Guía práctica. Madrid: OMT.

Organización Mundial del Turismo. (2016). Alianza entre turismo y cultura en el Perú - Modelos de colaboración entre turismo, cultura y comunidad. Madrid: OMT.

Organización Mundial del Turismo. (2017). Declaración de Chengdu sobre el turismo y los Objetivos de Desarrollo Sostenible. OMT. Recuperado de http://cf.cdn.unwto.org/sites/all/files/pdf/12898_chengu_declaration_ga22_final_sp_clean.pdf

Organización Mundial del Turismo. (s.f.). Ética, Cultura y Responsabilidad Social. OMT. Recuperado de http://ethics.unwto.org/es/ content/codigo-etico-mundial-para-el-turismo

World Tourism Organization. (2011). Communicating Heritage. A Handbook for the Tourism Sector. Madrid: UNWTO.

World Tourism Organization. (2013a). Turismo y patrimonio cultural inmaterial. Madrid: OMT.

World Tourism Organization. (2013b). Tourism at World Heritage Sites. Challenges and opportunities. Madrid: UNWTO.

World Tourism Organization. (2015a). About UNWTO. UNWTO. Recuperado de http://cf.cdn.unwto.org/sites/all/files/docpdf/ aboutunwtoenoctober2015web.pdf

World Tourism Organization. (2015b). Global Report on Cultural Routes and Itineraries. Madrid: UNWTO.

World Tourism Organization. (2016). UNWTO Basic Documents (Vol. I). Madrid: UNWTO, Recuperado de http://cf.cdn.unwto.org/sites/ all/files/docpdf/130718basicdocumentsenweb.pdf

World Tourism Organization. (2017). Executive Summary of Discussion Paper on Sustainable Tourism for Development. UNWTO. Recuperado de http://cf.cdn.unwto.org/sites/all/files/pdf/a22_10_i_b_report_on_the_international_year_of_sustainable_tourism_for _development_2017_sp.pdf

World Tourism Organization. (2018a). Tourism and culture synergies. Madrid: UNWTO. Recuperado de https://www.e-unwto.org/doi/ pdf/10.18111/9789284418978

World Tourism Organization. (2018b). Tourism and Development. Madrid: UNWTO.

Juan Carlos Paredes Izquierdo

Director del Instituto de Investigación de la Escuela Profesional de Turismo y Hotelería de la Universidad de San Martín de Porres. Es director de la revista Turismo y Patrimonio y ha sido director de dos escuelas universitarias de turismo. Es graduado de la Facultad de Derecho y Ciencias Políticas de la Universidad de Lima y cuenta con una maestría en Gestión Cultural, Patrimonio y Turismo de la Universidad de San Martín de Porres.

jcparedesizquierdo@gmail.com 